\title{
Spin-Valley Qubit Dynamics in Exchange-Coupled Silicon Quantum Dots
}

\author{
Donovan Buterakos* and Sankar Das Sarma \\ Department of Physics, Condensed Matter Theory Center and Joint Quantum Institute, University of Maryland, \\ College Park, Maryland 20742-4111, USA
}

(Received 11 June 2021; accepted 23 November 2021; published 23 December 2021)

\begin{abstract}
The presence of valley states is a significant obstacle to realizing quantum information technologies in silicon quantum dots, as leakage into alternate valley states can introduce errors into the computation. We use a perturbative analytical approach to study the dynamics of exchange-coupled quantum dots with valley degrees of freedom. We show that if the valley splitting is large and electrons are not properly initialized to valley eigenstates, then the time evolution of the system will lead to spin-valley entanglement. Spin-valley entanglement will also occur if the valley splitting is small and electrons are not initialized to the same valley state. Additionally, we show that for small valley splitting, spin-valley entanglement does not affect the measurement probabilities of two-qubit systems; however, systems with more qubits will be affected. This means that two-qubit gate fidelities measured in two-qubit systems may miss the effects of valley degrees of freedom. Our work shows how the existence of valleys may adversely affect multiqubit fidelities even when the system temperature is very low. Although this is not an immediate problem in $\mathrm{Si}$ qubits, because the current focus is on controlling individual qubits, our work points to a possible future issue in many-qubit Si circuits.
\end{abstract}

DOI: 10.1103/PRXQuantum.2.040358

\section{INTRODUCTION}

Silicon quantum dots have been shown to be a promising candidate system for the realization of quantum information technologies due to their long coherence times, fast gate times, potential for scalability, and integration within the current semiconductor industry. Significant progress has been made in the study of Si-based quantum-dot-type qubits using either Si-MOS or Si-Ge devices, including the representative (but by no means exhaustive) recent experimental publications in Refs. [1-16]. An eventual large-scale quantum computer will solve problems that no classical digital computers can. One such problem, which ushered in the modern era of quantum computing, is the Shor algorithm for prime factorization [17]. This is a problem of great technological interest, as it is used in all modern cryptography. If one tries to factorize a 1000-digit number-which classical computers cannot, since the computation cost is exponential in the number of digits - one may need roughly a million logical qubits. Since quantum error correction is essential for quantum computing to actually work, each logical qubit

*dbuterak@umd.edu

Published by the American Physical Society under the terms of the Creative Commons Attribution 4.0 International license. Further distribution of this work must maintain attribution to the author(s) and the published article's title, journal citation, and DOI. may easily require several thousand physical qubits for its realization, so in the end a hypothetical quantum CPU trying to decisively beat classical computers in doing prime factorization may require on the order of $10^{10}$ physical qubits. Although this sounds like a huge number of qubits, an ordinary CPU chip today may host $10^{10}$ transistors or bits. Each of these transistors or classical bits is made of silicon, giving $\mathrm{Si}$ a huge materials and technological advantage in building a quantum computer. In this context, it is understandable why there are multiple large groups all over the world (e.g., INTEL, Princeton, Wisconsin, Sandia, Delft, Sydney, Hefei, etc.) involved in developing Si-based qubits, although in terms of the number of working qubits today, the Si system is well behind ion-trap or superconducting transmon qubits. So far, only between one and four Si qubits have been successfully demonstrated in the laboratory.

The physical property being used to create the Si qubit is the electron spin localized within an effective Si quantum dot near a $\mathrm{Si}$ surface or interface with another material, such as the $\mathrm{Si}_{-} \mathrm{SiO}_{2}$ MOS system or the Si-Ge twodimensional electron system. Since electron spin is by definition a quantum two-level system, an isolated localized electron spin, if it can be manipulated without much decoherence, is an ideal qubit. Silicon has an enormous advantage because electron spins in $\mathrm{Si}$ are relatively long lived with long spin coherence times even for natural $\mathrm{Si}$, which can be enhanced greatly by isotopic purification [18]. In addition, two-qubit gates can be implemented by 
the exchange coupling of neighboring localized spins by electrically controlling the tunnel coupling between neighboring quantum dots, allowing very fast gate operations. Thus, the long spin coherence time, the fast electrostatic gating, and the existing Si chip technology allowing scaling up in principle make Si-based quantum information processing extremely attractive. There is, however, one serious fundamental problem: the valley degree of freedom. Bulk Si has six equivalent conduction-band minima, with the ground state of Si quantum dots having two valleys that are energetically degenerate in the ideal limit. Thus, the ground-state Si quantum-dot electron spin can in principle be any of the four degenerate spin-valley states. Typically, there is always some valley splitting associated with the surface or interface $[19,20]$ but the magnitude of this valley splitting is uncontrollable and it varies randomly from dot to dot in an essentially random manner. The valley problem in silicon quantum dots - which has received little attention in the literature so far, although most researchers in the field recognize its importance [18-25] - is the topic of the current theoretical work.

The Si valley problem has mostly been considered in the context of the valley splitting in the quantum-dot qubit being large compared with the qubit temperature (approximately $25-100 \mathrm{mK}$ ) so that the thermal occupancy of the higher valley states remains negligible, enabling a valid two-level quantum description of the system in terms of only the electron spin states. This is indeed a serious potential problem, as the qubit can no longer be defined if higher valley states are occupied. Therefore, much work has gone into producing Si qubits with large valley splittings through materials development and improved fabrication as well as on characterizing valley splittings in individual quantum dots [26-33]. However, this may not be the only problem when two-qubit gate operations are carried out using interqubit exchange coupling. We find that the relative value of the valley splitting with respect to the exchange coupling becomes an important limiting factor even at $T=0$ when any valley splitting is, by definition, much larger than the temperature. In particular, we find that the valley splitting must be much larger than the interqubit exchange coupling to avoid leakage (i.e., quantum decoherence) for the $\mathrm{Si}$ system to operate as a multiqubit quantum computing platform and we also find that even when the valley splitting is large, the initialization of the valley states becomes a crucial consideration in multiqubit gate operations. This problem of valley splitting in the context of multiqubit gate operations as determined by the exchange coupling between quantum dots has not attracted attention yet in the literature, perhaps because of two reasons: (1) there are very few reports of two-qubit exchange gate operations in Si quantum-dot qubits; (2) and the currently achieved values for the exchange coupling are very small, so that the condition of a valley splitting being larger than the exchange energy is automatically satisfied when the valley splitting is larger than the temperature. Since the speed of the two-qubit gate operations is determined by the exchange coupling strength, a higher exchange coupling strength is desirable in the future for progress in Si-based quantum computing and we wish to alert the community to the fact that the valley-splitting issue is fundamental to two-qubit gate operations as a totally distinct problem from the one involving thermal occupancies of higher valley states.

The problem with having multiple valley states is that their presence can lead to leakage out of the computational space if the valley degeneracy is not adequately broken. Of the six valley states present in bulk silicon, four of these decouple when strain is applied to the sample but the degeneracy of the final two valley states is broken only by a small-valley-splitting term that is dependent on the microscopics of the system [34]. We emphasize that there is no known way to control this valley splitting in specific qubits and, in fact, one can only figure out the size of the valley splitting a posteriori. There are no existing in situ sample diagnostics providing the valley-splitting information for the working qubits beforehand. In the current work, we analyze the effect of valleys on the coupled qubit dynamics, finding that valleys are a much bigger problem for coupled qubits than has been realized so far and that the valley problem worsens radically as the number of qubits goes beyond two. In fact, we believe that the Si quantum computing community would be well advised to consider the valley problem now, before building circuits with tens of coupled quantum dots and finding out that they do not work because of the valley problem. The problem of principle that we have uncovered here can be "fixed" by having small exchange coupling (« valley splitting) but this means that the two-qubit gate operations will remain bounded by the valley-splitting energies.

In this paper, we use a Hubbard model to determine the dynamics of a system of two exchange-coupled quantum dots. We show that spin-valley entanglement can easily arise from time evolution of the system, which is detrimental to the use of the system for quantum information applications. This can be avoided if the valley splitting is large and electrons can be initialized to valley eigenstates and, in fact, we show that under these conditions the system maintains coherence and is unaffected to leading order by the valley degree of freedom. If any electron begins in a superposition of valley states, then spin-valley entanglement will result, but if the valley splitting is sufficiently large, electrons can be properly initialized to the valley ground state, avoiding this situation. However, if the valley splitting is small compared to the exchange interaction strength, then an undesirable spin-valley entanglement is more difficult to avoid, as it will be present unless all electrons are initialized to the same valley state, which is generally difficult to achieve without a large valley splitting. 
Additionally, we show that when the valley splitting is small, the measurement probabilities of a two-qubit system are unaffected and are identical to the corresponding measurement probabilities in an ideal system without valley degrees of freedom. However, this is not the case in systems with more than two qubits and we give examples of gate sequences that give different measurement probabilities in a system with valley states than in an ideal system. Because valley degeneracy affects larger systems but not two-qubit systems, two-qubit gate fidelities measured in two-qubit systems may not accurately account for the effects of valley states, as these effects are only observable in larger system sizes containing more than just two qubits. This last property that we discover has not been mentioned in the literature at all and there has been a feeling that if the two-qubit gates work, the valleydegeneracy problem is irrelevant. We show that this is false - one could have perfectly working two-qubit gates but the system will lose quantum information through spinvalley entanglement as one scales up to more qubits. This is a very serious issue that requires resolution before more qubits are added to the circuit. We work at $T=0$ throughout, so that the well-understood problem of the thermal occupancy of higher valley states is a nonissue. We consider the valley-degeneracy question only in the context of gate operations driven by the interdot exchange coupling. Just to avoid any misunderstanding, our definition of "large" and "small" valley splitting is as compared with the interdot exchange coupling and not as compared with the temperature, as we are at $T=0$. Thus, the valley splitting could be large (or small) for our two-qubit considerations even if it is considered to be small (or large) for thermal occupancies that are relevant for single-qubit operations. For example, when the exchange coupling is large, as is necessary for fast two-qubit gate operations, the valley splitting for our consideration is small and independent of temperature.

This paper is organized as follows. In Sec. II, we present our model and give the Hamiltonian that we use. In Sec. III, we diagonalize the Hamiltonian for a system of two electrons in two quantum dots, for both triplet and singlet spin configurations. In Sec. IV, we discuss the dynamics of the two-qubit system, first for the case where the valley splitting is large and then also for the case of small or zero valley splitting. In Sec. V, we give examples of gate sequences for which valley effects are observable in four qubit systems, even when they are not detectable in similar two-qubit systems. We give our conclusions in Sec. VI.

\section{MODEL AND HAMILTONIAN}

The Fermi surface of silicon contains six electron pockets, leading to a sixfold degeneracy in the band-structure minima. By applying tensile strain to the sample, two of these valleys are energetically separated from the other four but remain nearly degenerate to each other. Therefore, in addition to its spin, an electron in a silicon quantum dot will contain a valley quantum number denoting whether it fills the $|+z\rangle$ or $|-z\rangle$ valley state. Microscopic features of the system introduce a small position-dependent valley splitting. We consider a double quantum dot in silicon, which yields the following effective Hubbard Hamiltonian [34-36]:

$$
\begin{aligned}
H= & \sum_{s \in\{\uparrow, \downarrow\}} \sum_{j=1}^{2}\left(\tilde{\Delta}_{j} c_{j,+z, s}^{\dagger} c_{j,-z, s}+\tilde{\Delta}_{j}^{*} c_{j,-z, s}^{\dagger} c_{j,+z, s}\right) \\
& +\frac{\epsilon}{2}\left(n_{1}-n_{2}\right)+t_{c} \sum_{s \in\{\uparrow, \downarrow\}} \sum_{v= \pm z}\left(c_{1, v, s}^{\dagger} c_{2, v, s}+c_{2, v, s}^{\dagger} c_{1, v, s}\right) \\
& +\sum_{j=1}^{2} \frac{U}{2} n_{j}\left(n_{j}-1\right)
\end{aligned}
$$

where $c_{j, v, s}$ is the second quantized annihilation operator for dot $j$, valley state $v$, and spin $s$ and where $n_{j}=$ $n_{j,+z, \uparrow}+n_{j,+z, \downarrow}+n_{j,-z, \uparrow}+n_{j,-z, \downarrow}$. Here, $\epsilon$ is the detuning between the two quantum dots, $t_{c}$ is the tunneling constant between the two dots, and $\tilde{\Delta}_{j}$ determines the valley splitting of $\operatorname{dot} j$. We define $U$ to be the Coulombenergy difference between a dot occupied by two electrons and a state with one electron occupying each dot. Thus we use a short-range Coulomb interaction term that is independent of valley states (the inclusion of an explicit valley dependence in the interaction complicates the calculation but does not affect our conclusion). Let $\tilde{\Delta}_{j}=\Delta_{j} e^{-i \phi_{j}}$, where $\Delta_{j}=\left|\tilde{\Delta}_{j}\right|$. Define $\left|j_{ \pm}\right\rangle=(|j,+z\rangle \pm$ $\left.e^{i \phi_{j}}|j,-z\rangle\right) / \sqrt{2}$. Here, $\left|j_{ \pm}\right\rangle$are the eigenstates of a single electron in a single dot $j$. Then, for a single electron in a double quantum dot, transforming $H$ into the basis $\left\{\left|1_{+}\right\rangle,\left|1_{-}\right\rangle,\left|2_{+}\right\rangle,\left|2_{-}\right\rangle\right\}$gives the following:

$$
H=\left(\begin{array}{cccc}
\epsilon / 2+\Delta_{1} & 0 & t_{+} & t_{-} \\
0 & \epsilon / 2-\Delta_{1} & t_{-} & t_{+} \\
t_{+}^{*} & t_{-}^{*} & -\epsilon / 2+\Delta_{2} & 0 \\
t_{-}^{*} & t_{+}^{*} & 0 & -\epsilon / 2-\Delta_{2}
\end{array}\right)
$$

where $t_{ \pm}=\left(1 \pm e^{i \delta \phi}\right) t_{c} / 2$ and $\delta \phi=\phi_{2}-\phi_{1}$. By this definition, $t_{+}$is the tunneling coefficient between the valley ground states of both dots $\left(\left|1_{+}\right\rangle\right.$to $\left.\left|2_{+}\right\rangle\right)$or between the valley excited states of both dots $\left(\left|1_{-}\right\rangle\right.$to $\left.\left|2_{-}\right\rangle\right)$. $t_{-}$is the tunneling coefficient between the valley ground state of one dot and the valley excited state of the other $\left(\left|1_{+}\right\rangle\right.$to $\left|2_{-}\right\rangle$or $\left|1_{-}\right\rangle$to $\left.\left|2_{+}\right\rangle\right)$. Equation (2) is equivalent to the twodot Hamiltonian given in Ref. [34] up to a constant energy shift. We emphasize that this Hubbard-model Hamiltonian describes quantum-dot qubit coupling in reasonably realistic situations [35-37]. 


\section{DIAGONALIZATION OF HAMILTONIAN}

In order to determine the effects of valley degeneracy and splitting on the exchange coupling between two dots, we consider two electrons in this two-dot system, diagonalizing the resulting Hamiltonian. We do this separately for the case when the electrons form a spin triplet and when they form a spin singlet.

\section{A. Triplet spin configuration}

Consider two electrons with a triplet spin configuration. Then, due to the Pauli exclusion principle, the electrons must occupy different orbital or valley states. We assume that $U \gg t_{c}, \Delta_{j}, \epsilon$, as the short-range Coulomb interaction energy is the largest energy scale in the system. Then, there are four low-energy states: $\left|1_{+} 2_{+}\right\rangle,\left|1_{+} 2_{-}\right\rangle,\left|1_{-} 2_{+}\right\rangle$, and $\left|1_{-} 2_{-}\right\rangle$(note that because we are working entirely within the triplet subspace, we denote states only by their orbital and valley parts, with the understanding that the spin part forms a triplet; explicit definitions of these states are given in the Appendix). These states couple via tunneling to the two high-energy states, $\left|1_{+} 1_{-}\right\rangle$and $\left|2_{+} 2_{-}\right\rangle$, where the electrons occupy both valley states in a single dot. Perturbation theory to first order in $U^{-1}$ gives the following Hamiltonian for the four lowest-energy states after including the effects of coupling to the two high-energy states:

$$
H_{T}=\left(\begin{array}{cc}
\Delta_{1}+\Delta_{2}-\frac{2\left|t_{-}\right|^{2}}{U} & \frac{t_{-} t_{+}^{*}-t_{+} t_{-}^{*}}{U} \\
\frac{t_{+} t_{-}^{*}-t_{-} t_{+}^{*}}{U} & \Delta_{1}-\Delta_{2}-\frac{2\left|t_{+}\right|^{2}}{U} \\
\frac{t_{-} t_{+}^{*}-t_{+} t_{-}^{*}}{U} & \frac{2\left|t_{+}\right|^{2}}{U} \\
\frac{2\left|t_{-}\right|^{2}}{U} & \frac{t_{+}+t_{-}^{*}-t_{-} t_{+}^{*}}{U}
\end{array}\right.
$$

$$
\left.\begin{array}{cc}
\frac{t_{+} t_{-}^{*}-t_{-} t_{+}^{*}}{U} & \frac{2\left|t_{-}\right|^{2}}{U} \\
\frac{2\left|t_{+}\right|^{2}}{U} & \frac{t_{-} t_{+}^{*}-t_{+} t_{-}^{*}}{U} \\
-\Delta_{1}+\Delta_{2}-\frac{2\left|t_{+}\right|^{2}}{U} & \frac{t_{+} t_{-}^{*}-t_{-} t_{+}^{*}}{U} \\
\frac{t_{-} t_{+}^{*}-t_{+} t_{-}^{*}}{U} & -\Delta_{1}-\Delta_{2}-\frac{2\left|t_{-}\right|^{2}}{U}
\end{array}\right),
$$

The details of the derivation of Eq. (3) are given in the Appendix. Note that to leading order in $\epsilon / U$, the four lowenergy states are independent of $\epsilon$. This is because $\epsilon$ only affects the energies of states where both electrons occupy the same dot and these are already energetically separated from the other states by the large on-site Coulomb interaction strength $U$ and hence do not contribute in the leading order. If $\epsilon$ is allowed to be of the same scale as $U$, as is done in some experiments to control the exchange interaction, then these results must be adjusted accordingly. Specifically, where $U$ appears in the denominator of terms in Eq. (3) it must be replaced with $U \pm \epsilon$, depending on which state introduces each term. However, for the purpose of this paper, we focus on the situation where $\epsilon \ll U$, as is the case in experiments that use barrier control of the exchange interaction. In order to further study the system dynamics, Eq. (3) must be fully diagonalized. Since this cannot be easily analytically done for arbitrary $\Delta_{j}$ and $t_{c}$, we instead consider two different limits for small and large $\Delta_{j}$. In the limit where $\Delta_{j} \gg t_{c}^{2} / U$, the matrix in Eq. (3) is already diagonalized to leading order in $t_{c}^{2} / U \Delta_{j}$ and the energies are given by its diagonal entries. In the limit where $\Delta_{j} \ll t_{c}^{2} / U$, diagonalizing Eq. (3) yields the following energies:

$$
\begin{aligned}
& E_{1}=-\frac{4 t_{c}^{2}}{U}, \\
& E_{2}=-\left|\tilde{\Delta}_{1}+\tilde{\Delta}_{2}\right| \\
& E_{3}=0 \\
& E_{4}=\left|\tilde{\Delta}_{1}+\tilde{\Delta}_{2}\right|=\sqrt{\Delta_{1}^{2}+\Delta_{2}^{2}+2 \Delta_{1} \Delta_{2} \cos \delta \phi} .
\end{aligned}
$$

The corresponding eigenstates are given by

$$
\begin{aligned}
\left|\psi_{1}\right\rangle= & \frac{1}{\sqrt{2} t_{c}}\left(t_{-}\left|1_{+} 2_{+}\right\rangle-t_{+}\left|1_{+} 2_{-}\right\rangle+t_{+}\left|1_{-} 2_{+}\right\rangle-t_{-}\left|1_{-} 2_{-}\right\rangle\right), \\
\left|\psi_{2}\right\rangle= & {\left[\frac{\left(\Delta_{2}+\Delta_{1}\right) t_{+}}{2 t_{c}\left|\tilde{\Delta}_{1}+\tilde{\Delta}_{2}\right|}\left(\left|1_{+} 2_{+}\right\rangle+\left|1_{-} 2_{-}\right\rangle\right)+\frac{\left(\Delta_{2}-\Delta_{1}\right) t_{-}}{2 t_{c}\left|\tilde{\Delta}_{1}+\tilde{\Delta}_{2}\right|}\left(\left|1_{+} 2_{-}\right\rangle+\left|1_{-} 2_{+}\right\rangle\right)\right.} \\
& \left.-\frac{1}{2 t_{c}}\left(t_{+}\left|1_{+} 2_{+}\right\rangle-t_{-}\left|1_{+} 2_{-}\right\rangle+t_{-}\left|1_{-} 2_{+}\right\rangle-t_{+}\left|1_{-} 2_{-}\right\rangle\right)\right],
\end{aligned}
$$




$$
\begin{aligned}
\left|\psi_{3}\right\rangle= & \frac{1}{\sqrt{2} t_{c}\left|\tilde{\Delta}_{1}+\tilde{\Delta}_{2}\right|}\left[\left(\Delta_{2}-\Delta_{1}\right) t_{-}\left(\left|1_{+} 2_{+}\right\rangle+\left|1_{-} 2_{-}\right\rangle\right)+\left(\Delta_{2}+\Delta_{1}\right) t_{+}\left(\left|1_{+} 2_{-}\right\rangle+\left|1_{-} 2_{+}\right\rangle\right)\right], \\
\left|\psi_{4}\right\rangle= & {\left[\frac{\left(\Delta_{2}+\Delta_{1}\right) t_{+}}{2 t_{c}\left|\tilde{\Delta}_{1}+\tilde{\Delta}_{2}\right|}\left(\left|1_{+} 2_{+}\right\rangle+\left|1_{-} 2_{-}\right\rangle\right)+\frac{\left(\Delta_{2}-\Delta_{1}\right) t_{-}}{2 t_{c}\left|\tilde{\Delta}_{1}+\tilde{\Delta}_{2}\right|}\left(\left|1_{+} 2_{-}\right\rangle+\left|1_{-} 2_{+}\right\rangle\right)\right.} \\
& \left.+\frac{1}{2 t_{c}}\left(t_{+}\left|1_{+} 2_{+}\right\rangle-t_{-}\left|1_{+} 2_{-}\right\rangle+t_{-}\left|1_{-} 2_{+}\right\rangle-t_{+}\left|1_{-} 2_{-}\right\rangle\right)\right] .
\end{aligned}
$$

\section{B. Singlet spin configuration}

We now repeat the same calculation for a pair of electrons in a singlet spin configuration. In this case, the same six orbital states as in the triplet case are present, with the addition of four doubly occupied states $\left|1_{+1 \downarrow}\right\rangle,\left|1_{-1 \downarrow}\right\rangle,\left|2_{+\mid l}\right\rangle$, and $\left|2_{-1 l}\right\rangle$, since both electrons can occupy the same valley and orbital state. As above, we apply perturbation theory in $U^{-1}$ and calculate the Hamiltonian for the four lowest-energy states, yielding:

$$
H_{S}=\left(\begin{array}{cccc}
\Delta_{1}+\Delta_{2}-\frac{2\left|t_{-}\right|^{2}+4\left|t_{+}\right|^{2}}{U} & -\frac{2 t_{-} t_{+}^{*}}{U} & -\frac{2 t_{+}+t_{-}^{*}}{U} & -\frac{2\left|t_{-}\right|^{2}}{U} \\
-\frac{2 t_{+}+t_{-}^{*}}{U} & \Delta_{1}-\Delta_{2}-\frac{2\left|t_{+}\right|^{2}+4\left|t_{-}\right|^{2}}{U} & -\frac{2\left|t_{+}\right|^{2}}{U} & -\frac{2 t_{-} t_{+}^{*}}{U} \\
-\frac{2 t_{-}-t_{+}^{*}}{U} & -\frac{2\left|t_{+}\right|^{2}}{U} & -\Delta_{1}+\Delta_{2}-\frac{2\left|t_{+}\right|^{2}+4\left|t_{-}\right|^{2}}{U} & -\frac{2 t_{+} t_{-}^{*}}{U} \\
-\frac{2\left|t_{-}\right|^{2}}{U} & -\frac{2 t_{+} t_{-}^{*}}{U} & -\frac{2 t_{-} t_{+}^{*}}{U} & -\Delta_{1}-\Delta_{2}-\frac{2\left|t_{-}\right|^{2}+4\left|t_{+}\right|^{2}}{U}
\end{array}\right) .
$$

The details of the derivation of Eq. (6) are again given in the Appendix. Diagonalizing this matrix in the limit where $\Delta_{j} \ll t_{c}^{2} / U$ gives the following energies:

$$
\begin{aligned}
& E_{1}=0 \\
& E_{2}=-\frac{4 t_{c}^{2}}{U}-\left|\tilde{\Delta}_{1}+\tilde{\Delta}_{2}\right|, \\
& E_{3}=-\frac{4 t_{c}^{2}}{U} \\
& E_{4}=-\frac{4 t_{c}^{2}}{U}+\left|\tilde{\Delta}_{1}+\tilde{\Delta}_{2}\right| .
\end{aligned}
$$

The corresponding singlet-case eigenstates are precisely the same as for the triplet case given by Eq. (5). Note that $E_{2}, E_{3}$, and $E_{4}$ are less than the corresponding triplet energies; however, $E_{1}$ is greater than the corresponding triplet energy. In each case, the difference is $\pm J_{0}$, where $J_{0}=4 t_{c}^{2} / U$ is the strength of the exchange interaction in an ideal system that does not have any valley degeneracy.

\section{SYSTEM DYNAMICS}

We now investigate the dynamics of a system prepared in a specific initial state and allowed to evolve under the Hamiltonian for some time $t$. This corresponds to the coupled qubit dynamics under gate operation, which controls quantum computation. We show that when the initial state is not prepared with each electron in the same valley state, leakage between valley states will introduce error. If the valley splitting $\Delta_{j}$ is small, this error can occur even if all electrons are initialized in the valley ground states $\left|j_{-}\right\rangle$if the phases $\phi_{j}$ differ from one another. We first examine the large valley-splitting limit where $\Delta_{j} \gg J_{0}$, followed by the small valley-splitting limit where $\Delta_{j} \ll J_{0}$.

\section{A. Large valley splitting}

When $\Delta_{j} \gg t_{c}^{2} / U$, the system dynamics are determined to leading order by the diagonal entries of Eqs. (3) and (6). The off-diagonal elements only affect the energies to order $J_{0}^{2} / \Delta_{j}$. If the system is prepared in one of the valley eigenstates, there will be an effective exchange interaction $J_{+}=4\left|t_{+}\right|^{2} / U$ for valley states $\left|1_{+} 2_{+}\right\rangle$and $\left|1_{-} 2_{-}\right\rangle$, and an effective exchange interaction $J_{-}=4\left|t_{-}\right|^{2} / U$ for valley states $\left|1_{+} 2_{-}\right\rangle$and $\left|1_{-} 2_{+}\right\rangle$. Thus, to leading order in $J_{0} / \Delta_{j}$, the presence of energetically separated valley states does not affect the dynamics of the system as long as the initial state is a valley eigenstate.

Since valley eigenstates have trivial dynamics in this regime, we consider a system not initialized to a valley eigenstate, representing a situation where some valley error has occurred during initialization. In such a scenario, the time evolution of the system can give rise to spin-valley entanglement, which will propagate the error to the spin state of the system. Consider, for example, an initial state where qubit 1 starts in the $\left|1_{+\uparrow}\right\rangle$ state, but qubit 2 starts in the state $\left(\left|2_{+\downarrow}\right\rangle+\left|2_{-\downarrow}\right\rangle\right) / \sqrt{2}$. Then, after some time $t$, the system will evolve to

$$
\begin{aligned}
|\Psi(t)\rangle= & \frac{1}{2}\left|1_{+} 2_{+}\right\rangle\left(\left|T_{0}\right\rangle+e^{i J_{+} t}|S\rangle\right) \\
& +\frac{1}{2} e^{i t\left\{2 \Delta_{2}+\left[\left(J_{+}-J_{-}\right) / 2\right]\right\}}\left|1_{+} 2_{-}\right\rangle\left(\left|T_{0}\right\rangle+e^{i J_{-} t}|S\rangle\right),
\end{aligned}
$$


where $\left|T_{0}\right\rangle$ and $|S\rangle$ are the triplet and singlet spin states, respectively. Suppose, for simplicity, that $\phi_{1}=\phi_{2}$, and thus $J_{-}=0$ and $J_{+}=J_{0}$. Then, the system reaches a maximally entangled state when $J_{0} t=(2 k+1) \pi$ for integer $k$ :

$$
\begin{aligned}
\mid \Psi( & \left.\left(\frac{(k+1) \pi}{J_{0}}\right)\right\rangle \\
= & \frac{1}{\sqrt{2}}\left|1_{+} 2_{+}\right\rangle|\downarrow \uparrow\rangle \\
& +\frac{1}{\sqrt{2}} e^{(2 k+1) \pi i\left[\left(2 \Delta_{2} / J_{0}\right)+(1 / 2) \cos ^{2}(\delta \phi / 2)\right]}\left|1_{+} 2_{-}\right\rangle|\uparrow \downarrow\rangle .
\end{aligned}
$$

Because the electron in dot 1 is initialized in a valley eigenstate in this example, it remains in that state throughout the evolution of the system. Thus, the state at any particular point in time corresponds a point on each of two Bloch spheres that represent the combined spin state (singlet or triplet) and the valley state of the second electron. This is not a one-to-one correspondence, as different entangled states can correspond to the same set of points; nevertheless, it is useful for visualizing the information stored in the spin and valley states and the entanglement between them.

In Fig. 1, we plot the path $|\Psi(t)\rangle$ traces on the two Bloch spheres. The path begins on the surface of both Bloch spheres, indicating that the initial state is separable. As time evolves to $t=\pi / J_{0}$, the path spirals toward the center of both Bloch spheres, indicating maximal spin-valley entanglement. In general, spin-valley entanglement is detrimental to quantum information applications, since
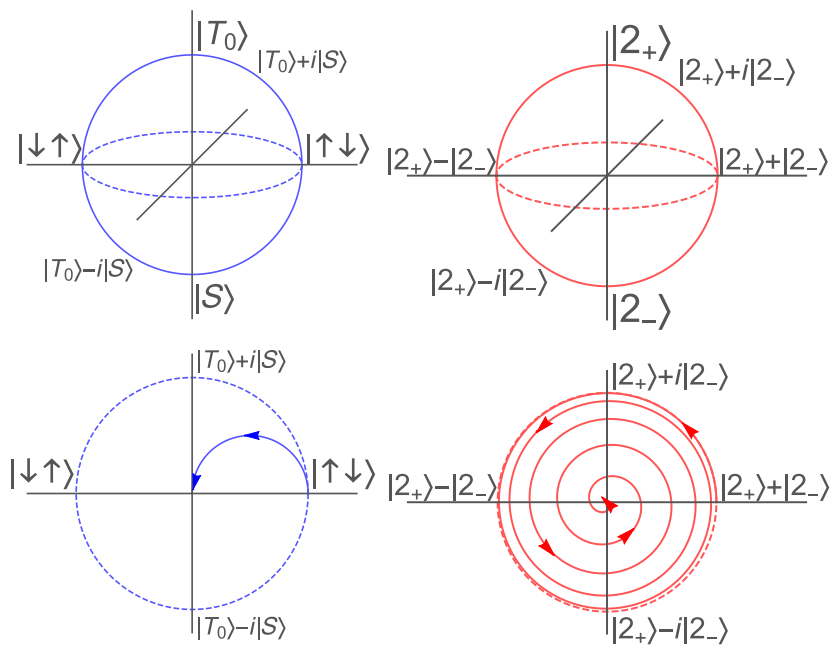

FIG. 1. Top: the Bloch-sphere representation of the spin qubit in the singlet-triplet basis and the valley qubit of the second dot (the first dot will always be in the valley state $\left|1_{+}\right\rangle$). Bottom: the time evolution of a state given by Eq. (8) for $t$ ranging from 0 to $\pi / J_{0}$, with $\Delta_{2}=5 J_{0}$ and $\phi_{1}=\phi_{2}$. The $x-y$ cross sections of the Bloch spheres are plotted since the state stays entirely within the $x-y$ planes. measuring the spin state without the ability to measure the corresponding valley state can result in a complete loss of qubit information. Thus it is imperative to initialize the system in valley eigenstates - otherwise, information will leak out unwittingly through spin-valley entanglement during the coupled qubit dynamical evolution.

\section{B. Small valley splitting}

We now consider a situation where the valley splitting is small compared to the exchange interaction strength. In this regime, the dynamics are dictated by the states and energies in Eqs. (3), (5), and (6). Because the singlet and triplet spin configurations share the same valley eigenstates, there is an effective exchange interaction $\pm J_{0}$ depending on the specific valley states occupied. We show that unless the system is initialized with all electrons occupying the same valley state, the time evolution of the coupled system will result in spin-valley entanglement. It is difficult to initialize the system in this way due to the near degeneracy of the valley states. Additionally, if the phase difference $\delta \phi$ between dots is nonzero, as is often the case, spin-valley entanglement will occur even if all electrons are initialized to their individual ground valley states. In fact, we are not aware of any experimental control capable of tuning the intervalley phase difference $\delta \phi$, which we see as a potential problem requiring resolution for Si spin qubits to work in a large circuit with many operational qubits.

To demonstrate the presence of spin-valley entanglement that can arise, consider an initial state $\left|1_{+\uparrow} 2_{+\downarrow}\right\rangle$. For simplicity, suppose that $\Delta_{1}=\Delta_{2}=\Delta$ and suppose that there is a nonzero phase difference $\delta \phi$. Then, after some time $t$, the state will evolve to

$$
\begin{aligned}
|\Psi(t)\rangle= & \frac{t_{-}^{*}}{2 t_{c}}\left|\psi_{1}\right\rangle\left(e^{i J_{0} t}\left|T_{0}\right\rangle+|S\rangle\right) \\
& +\frac{t_{+}^{*}}{2 \sqrt{2} t_{c}}\left[\left(\frac{t_{c}}{\left|t_{+}\right|}-1\right) e^{i\left(2\left|t_{+}\right| / t_{c}\right) \Delta t}\left|\psi_{2}\right\rangle\right. \\
& \left.+\left(\frac{t_{c}}{\left|t_{+}\right|}+1\right) e^{-i\left(2\left|t_{+}\right| / t_{c}\right) \Delta t}\left|\psi_{4}\right\rangle\right]\left(\left|T_{0}\right\rangle+e^{i J_{0} t}|S\rangle\right) .
\end{aligned}
$$

By definition, $2\left|t_{+}\right| / t_{c}=2|\cos (\delta \phi / 2)|$, which equals $\left|\tilde{\Delta}_{1}+\tilde{\Delta}_{2}\right| / \Delta$ when $\Delta_{1}=\Delta_{2}$. In Fig. 2, we plot the path on the Bloch sphere drawn out by total spin of $|\Psi(t)\rangle$. This path is independent of the value of $\Delta$ and forms an ellipse confined to the $x-y$ plane. The path given by the valley states, shown in Fig. 3, forms a rose curve that lies in the plane defined by $x+y \tan \delta \phi=1$. Its projection into the $x-y$ plane is a chord of the unit circle, the angle of which is independent of $\Delta$ and is determined solely by $\delta \phi$. The exact shape of the rose curve is given by the quantity $\Delta \cos (\delta \phi / 2) / J_{0}$. 


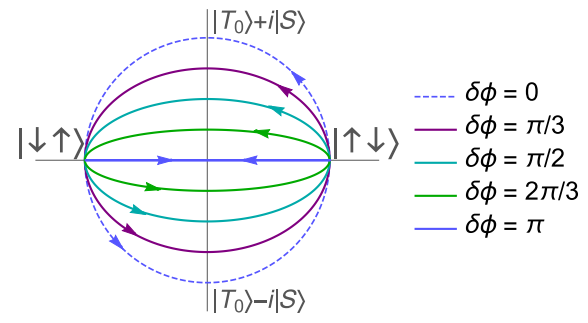

FIG. 2. The time evolution of the spin state given by Eq. (10) for $t$ ranging from 0 to $2 \pi / J_{0}$, plotted for different values of $\delta \phi$. The $x-y$ cross section of the Bloch sphere is shown since the path stays entirely within the $x-y$ plane. These paths are independent of $\Delta$.

In general, $|\Psi(t)\rangle$ is an entangled state, with two exceptions. $|\Psi(t)\rangle$ is separable if $\phi_{1}=\phi_{2}$, as this makes $t_{-}=0$, causing the first term in Eq. (10) to vanish. $|\Psi(t)\rangle$ also becomes separable when $t=k \pi / J_{0}$ (with integer $k$ ), as this causes the spin states in both terms of Eq. (10) to become identical. Conversely, $|\Psi(t)\rangle$ becomes a maximally entangled state when $\delta \phi=\pi$ and $J_{0} t=(2 k+1) \pi / 2$, which causes Eq. (10) to simplify to the following:

$$
\begin{aligned}
& \frac{1}{2 \sqrt{2}}\left(\left|1_{+} 2_{+}\right\rangle-\left|1_{-} 2_{-}\right\rangle\right)\left(i^{2 k+1}\left|T_{0}\right\rangle+|S\rangle\right) \\
& \quad+\frac{1}{2 \sqrt{2}}\left(\left|1_{+} 2_{+}\right\rangle+\left|1_{-} 2_{-}\right\rangle\right)\left(\left|T_{0}\right\rangle+i^{2 k+1}|S\rangle\right) .
\end{aligned}
$$

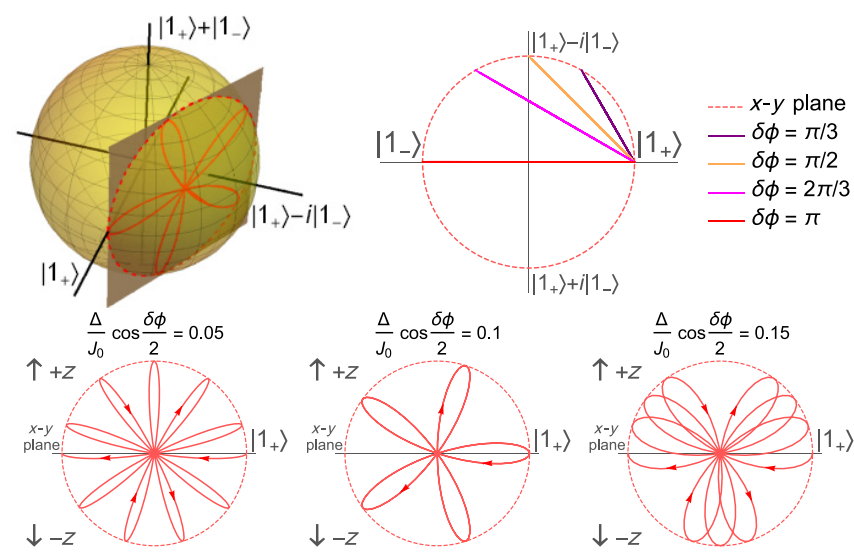

FIG. 3. Top left: the Bloch-sphere representation of the time evolution of the valley state of dot 1 in Eq. (10). The path forms a rose curve that lies within the plane given by $x+y \tan \delta \phi=1$. Top right: the projection of this path into the $x-y$ plane, plotted for different values of $\delta \phi$. The plot for the valley state of dot 2 is identical but reflected vertically. Bottom: sample rose curves plotted for values of the quantity $\Delta \cos (\delta \phi / 2) / J_{0}$ equal to 0.05 , 0.1 , and 0.15 . The horizontal axis is the chord plotted in the top right figure, and the dashed circle is the intersection of the Bloch sphere with the plane $x+y \tan \delta \phi=1$.
It is instructive to consider the probability of recovering the initial spin state $|\uparrow \downarrow\rangle$ when measuring the spin of $|\Psi(t)\rangle$ after some time (leaving the valley state unmeasured). From Eq. (10), this probability is found to be given by

$$
P\left(\left|1_{+\uparrow} 2_{+\downarrow}\right\rangle \rightarrow|\uparrow \downarrow\rangle\right)=\frac{1}{2}\left(1+\cos J_{0} t\right) .
$$

This is precisely the same measurement probability as would result from time evolution in an "ideal" one-valley system. Differences occur when measuring in a different basis; for example, the probability of obtaining the state $(|\uparrow \downarrow\rangle+i|\downarrow \uparrow\rangle) / \sqrt{2}$ is given by

$$
P\left(\left|1_{+\uparrow} 2_{+\downarrow}\right\rangle \rightarrow \frac{|\uparrow \downarrow\rangle+i|\downarrow \uparrow\rangle}{\sqrt{2}}\right)=\frac{1}{2}-\frac{1}{2} \cos ^{2} \frac{\delta \phi}{2} \sin J_{0} t .
$$

If $\phi_{1}=\phi_{2}$, meaning that the two electrons start in the same valley states, then Eq. (13) reduces to $\left(1-\sin J_{0} t\right) / 2$, which is the same result as would be given by an ideal system. However, if $\delta \phi=\pi$, then the two electrons start in opposite valley states and Eq. (13) reduces to $P=1 / 2$, independent of time. In this case, when $J_{0} t=(2 k+1) \pi / 2$, the probability outcome of measuring the spin in any basis gives $1 / 2$, as the spin state is maximally entangled with the valley state.

Despite the difference between Eq. (13) and the ideal case, spin-valley entanglement is difficult to observe in a system of two quantum dots. This is because it is difficult to directly measure in the basis containing the state $(|\uparrow \downarrow\rangle+i|\downarrow \uparrow\rangle) / \sqrt{2}$. Generally, if a quantum algorithm were to require such a measurement, the measurement would be performed by applying a $\sqrt{\text { SWAP }}$ gate and then measuring in the $z$ basis, which in an ideal system would produce the same result. However, $\sqrt{\mathrm{SWAP}}$ gates are performed via the exchange interaction, which can disentangle a spin-valley entangled state. In fact, for two qubits, as long as states are initialized and measured in the $z$ basis, valley splitting will not affect the measurement probability to first order in $\Delta / J_{0}$. This is because the eigenstates given by Eq. (5) simultaneously diagonalize both the triplet and singlet Hamiltonians, given in Eqs. (3) and (6). Additionally, the energy difference between the corresponding triplet and singlet energies, given in Eqs. (4) and (7), is $\pm J_{0}$ for every eigenstate. Simply measuring in the $z$ basis cannot distinguish between rotations by $+J_{0}$ or $-J_{0}$.

One possible way to make such a distinction between rotations is the following. Start with a state $\left|\psi_{1}\right\rangle$ and perform a partial rotation (perhaps $2 \pi / 3$ ) via the exchange interaction $J_{0}$. Then allow $\left|\psi_{1}\right\rangle$ through some method to be transformed to any of the other states $\left|\psi_{2}\right\rangle$ through $\left|\psi_{4}\right\rangle$. One way in which this might happen is to let the system precess under the valley splitting, but with no exchange 
interaction present (note that in this case, $\Delta \ll J_{0}$ does not hold). Finally, complete the initial rotation from the first step. In an ideal one-valley case, the system will have undergone one complete rotation. However, in a system with valley splitting, the last part of the rotation will be in the opposite direction to the first part and thus will not form a complete rotation. This will affect the measurement probabilities in the $z$ basis. However, it is not possible to complete the second step (rotating $\left|\psi_{1}\right\rangle$ into $\left|\psi_{2}\right\rangle$ ) in a system of two qubits while keeping $\Delta \ll J_{0}$ without adding additional terms to the Hamiltonian.

\section{OBSERVING VALLEY EFFECTS IN FOUR-QUBIT SYSTEMS}

In the previous discussion, we demonstrate that despite the spin-valley entanglement that occurs, the measurement probabilities in the $\{\uparrow, \downarrow\}$ basis are unaffected by the presence of valley states in a two-dot system. However, we now show that in a four-dot system, this is no longer the case. We do this by giving two examples of sequences of operations that will result in a different measurement probability in a two-valley system than the same operations would in an ideal one-valley system.

Consider the fully degenerate case where $\Delta_{1}=\Delta_{2}=0$ (exactly the same sequences apply when $\Delta_{j}$ are nonzero but we consider the degenerate case for the sake of simplicity). The time evolution of the exchange interaction can be used to perform $\sqrt{\text { SWAP }}$ gates, which swap both the spin and valley states of two electrons in adjacent dots. Consider an array of four quantum dots prepared in the initial state $\left|\uparrow_{-} \downarrow_{+} \uparrow_{+} \downarrow_{-}\right\rangle$(this state is equivalent to $\left|\uparrow_{+} \downarrow_{+} \uparrow_{+} \downarrow_{+}\right\rangle$where the phases $\phi_{1}=\phi_{4}=\pi$ ). Because the phases of $\Delta_{j}$ are not easy to control, the phases $\phi_{j}$ and the initial valley states of each electron are relatively random and thus there is no way to ensure that the system begins in the correct valley states. Thus, initial states such as those we consider are likely to occur and, as we show, will lead to errors in the computation. In this example, we only consider states with one electron confined to each dot and therefore we omit the dot numbers in our notation for the sake of notational brevity. Now perform the following operations to obtain $\left|\Psi_{\text {valley }}\right\rangle$ :

$$
\left|\Psi_{\text {valley }}\right\rangle=U_{23}^{\mathrm{SS}} U_{12}^{\mathrm{SS}} U_{34}^{\mathrm{SS}} U_{23}^{\mathrm{SS}}\left|\uparrow-\downarrow_{+} \uparrow+\downarrow_{-}\right\rangle,
$$

where $U_{i j}^{\mathrm{SS}}=\sqrt{\mathrm{SWAP}_{i j}}$ is the square root of SWAP gate between dots $i$ and $j$. This sequence of gates is depicted in Fig. 4 . Then, $\left|\Psi_{\text {valley }}\right\rangle$ can be explicitly obtained and is given by

$$
\begin{aligned}
\left|\Psi_{\text {valley }}\right\rangle= & \frac{1}{4}\left[\left|\uparrow_{+} \uparrow_{-} \downarrow_{+} \downarrow_{-}\right\rangle-i\left|\uparrow_{+} \uparrow-\downarrow_{-} \downarrow_{+}\right\rangle+2 i\left|\uparrow-\uparrow_{+} \downarrow_{+} \downarrow_{-}\right\rangle\right. \\
& +\left|\uparrow-\uparrow_{+} \downarrow_{-} \downarrow_{+}\right\rangle-\left|\uparrow_{+} \downarrow_{-} \uparrow_{-} \downarrow_{+}\right\rangle-i\left|\uparrow_{+} \downarrow_{+} \uparrow_{-} \downarrow_{-}\right\rangle-i\left|\uparrow-\downarrow_{-} \uparrow_{+} \downarrow_{+}\right\rangle \\
& +\left|\downarrow_{+} \uparrow-\downarrow_{-} \uparrow_{+}\right\rangle-i\left|\downarrow_{+} \downarrow_{-} \uparrow_{-} \uparrow_{+}\right\rangle+\left|\uparrow-\downarrow_{-} \downarrow_{+} \uparrow_{+}\right\rangle+i\left|\uparrow-\downarrow_{+} \downarrow_{-} \uparrow_{+}\right\rangle \\
& \left.+i\left|\downarrow_{+} \uparrow-\uparrow_{+} \downarrow_{-}\right\rangle+\left|\downarrow_{+} \uparrow_{+} \uparrow-\downarrow_{-}\right\rangle\right] .
\end{aligned}
$$

From this state, we calculate the measurement probability of the second dot being measured to be spin up, obtaining $P\left(2_{\uparrow}\right)=5 / 8$. Now consider the ideal one-valley case, where the same set of operations are performed on a system of four spins in the same initial spin configuration. Then, the resulting state $\left|\Psi_{\text {ideal }}\right\rangle$ will be given by

$$
\left|\Psi_{\text {ideal }}\right\rangle=\frac{1}{4}[(2+i)|\uparrow \uparrow \downarrow \downarrow\rangle-(1+2 i)|\uparrow \downarrow \uparrow \downarrow\rangle+|\downarrow \uparrow \downarrow \uparrow\rangle-i|\downarrow \downarrow \uparrow \uparrow\rangle+(1+i)|\uparrow \downarrow \downarrow \uparrow\rangle+(1+i)|\downarrow \uparrow \uparrow \downarrow\rangle] .
$$

In the ideal case, the probability of measuring the second spin to be up is $P\left(2_{\uparrow}\right)=1 / 2$, which is different from the case above where two valley states are present. This discrepancy arises from the fact that in the presence of valley states, there is a distinction between certain states that would be considered identical in the ideal case (e.g., $\left|\uparrow_{+} \uparrow_{-} \downarrow_{+} \downarrow_{-}\right\rangle$and $\left|\uparrow_{-} \uparrow_{+} \downarrow_{-} \downarrow_{+}\right\rangle$). This distinction prevents constructive or destructive interference between the states, which influences the final measurement probabilities (in the ideal case, both states are $|\uparrow \uparrow \downarrow \downarrow\rangle$, so their amplitudes should add together). This may have important implications for Si qubits. In order to perform correct calculations, all dots must be initialized to the same valley state and the opposite valley state should be considered a 


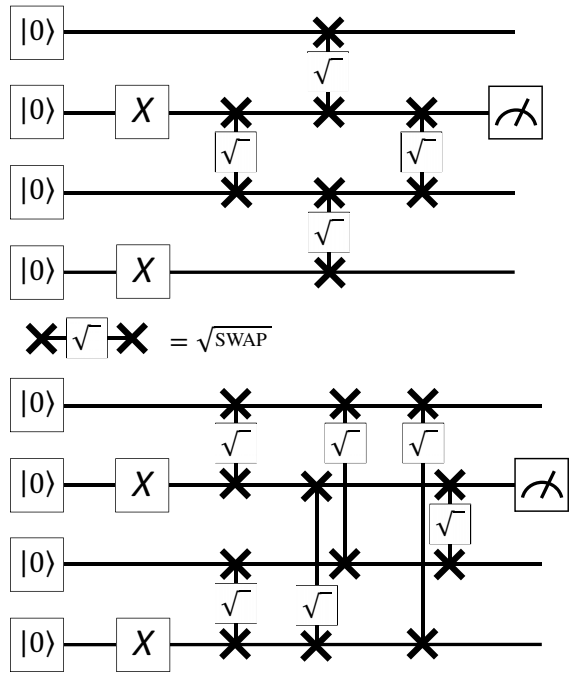

FIG. 4. Quantum circuits depicting the sequences of gates in Eqs. (14) (top) and (17) (bottom).

leakage state. The presence of a phase difference between dots $\delta \phi$ (leading to a $t_{-}$term) also introduces leakage into the system. However, the leakage states behave almost like the ideal states for small systems and, in fact, they are indistinguishable for systems of only two dots, as we show in the previous section. This makes errors resulting from leakage difficult to detect in small systems and small gate sequences. Thus fidelities of two-qubit gates measured in two-qubit systems may be measured as higher than their true value in multiqubit circuits, because these measurements cannot account for leakage into other valley states without applying a larger sequence of gates such as that of Eq. (14) to a larger number of dots.

The sequence of gates given in Eq. (14) is a short sequence of gates that yields a different measurement outcome in an ideal system than in a system with multiple valley states. This effect is present for any initial state where the electrons with the same spin do not all have the same valley state. Note that the initial state is dependent on the values of $\phi_{j}$ and the only way to control them is by controlling the valley phase. In Fig. 5, we plot the measurement probability of $\left|2_{\uparrow}\right\rangle$ versus the value of $\phi_{4}$, with $\phi_{1}=\phi_{2}=\phi_{3}=0$. When $\phi_{4}$ also equals 0 , the initial valley states of all electrons are the same and the measurement probability is the same as in the ideal case. As $\phi_{4}$ varies away from 0 , the measurement probability increases. This sequence of gates can be used to demonstrate the presence of valley-induced error, as if the state $\left|2_{\uparrow}\right\rangle$ is measured with probability greater than $1 / 2$, this is a result of valley-induced error. However, this sequence of gates cannot be used to show the converse in noisy systems, because if $\left|2_{\uparrow}\right\rangle$ is measured with probability $1 / 2$, the result is indistinguishable from noise-induced decoherence.

We now give a different sequence of gates that has a measurement probability of 1 in the ideal case and thus can be used to demonstrate initialization of electrons in the same valley state. Consider a ring of four quantum dots with the initial state $\left|\uparrow_{-} \downarrow_{+} \uparrow_{+} \downarrow_{-}\right\rangle$. Perform the following sequence of $\sqrt{\text { SWAP }}$ gates:

$$
\left|\Psi_{\text {valley }}\right\rangle=U_{23}^{\mathrm{SS}} U_{14}^{\mathrm{SS}} U_{13}^{\mathrm{SS}} U_{24}^{\mathrm{SS}} U_{12}^{\mathrm{SS}} U_{34}^{\mathrm{SS}}\left|\uparrow-\downarrow_{+} \uparrow+\downarrow_{-}\right\rangle .
$$

For this sequence of gates, $\left|\Psi_{\text {valley }}\right\rangle$ is given by

$$
\begin{aligned}
\left|\Psi_{\text {valley }}\right\rangle= & \frac{1}{4}\left[\left|\uparrow_{+} \uparrow_{-} \downarrow_{-} \downarrow_{+}\right\rangle-\left|\uparrow_{-} \uparrow_{+} \downarrow_{+} \downarrow_{-}\right\rangle+\left|\downarrow_{+} \downarrow_{-} \uparrow_{-} \uparrow_{+}\right\rangle-\left|\downarrow_{-} \downarrow_{+} \uparrow_{+} \uparrow_{-}\right\rangle\right. \\
& +\left|\uparrow_{+} \downarrow_{-} \downarrow_{+} \uparrow_{-}\right\rangle-\left|\uparrow_{-} \downarrow_{+} \downarrow_{-} \uparrow_{+}\right\rangle-\left|\downarrow_{+} \uparrow_{-} \uparrow_{+} \downarrow_{-}\right\rangle \\
& \left.+\left|\downarrow_{-} \uparrow_{+} \uparrow_{-} \downarrow_{+}\right\rangle+2\left|\downarrow_{+} \uparrow_{+} \downarrow_{-} \uparrow_{-}\right\rangle+2\left|\downarrow_{-} \uparrow_{-} \downarrow_{+} \uparrow_{+}\right\rangle\right] .
\end{aligned}
$$

The corresponding ideal state $\left|\Psi_{\text {ideal }}\right\rangle$ is simply

$$
\left|\Psi_{\text {ideal }}\right\rangle=|\downarrow \uparrow \downarrow \uparrow\rangle .
$$

With this sequence of gates, the ideal single-valley case will have measurement probabilities of $100 \%$ but the corresponding two-valley case will only have corresponding measurement probabilities of $75 \%$, simulating an unknown apparent decoherence although it arises simply from the inevitable spin-valley entanglement that is omnipresent in the multivalley qubits. In Fig. 5, we again plot the measurement probability of $\left|2_{\uparrow}\right\rangle$ versus the value of $\phi_{4}$ for this new gate sequence. When $\phi_{4}=0$, the measurement probability is $100 \%$, matching the ideal case, but for nonzero $\phi_{4}$ the measurement probability decreases, allowing the adverse effects of spin-valley entanglement to be detected. 

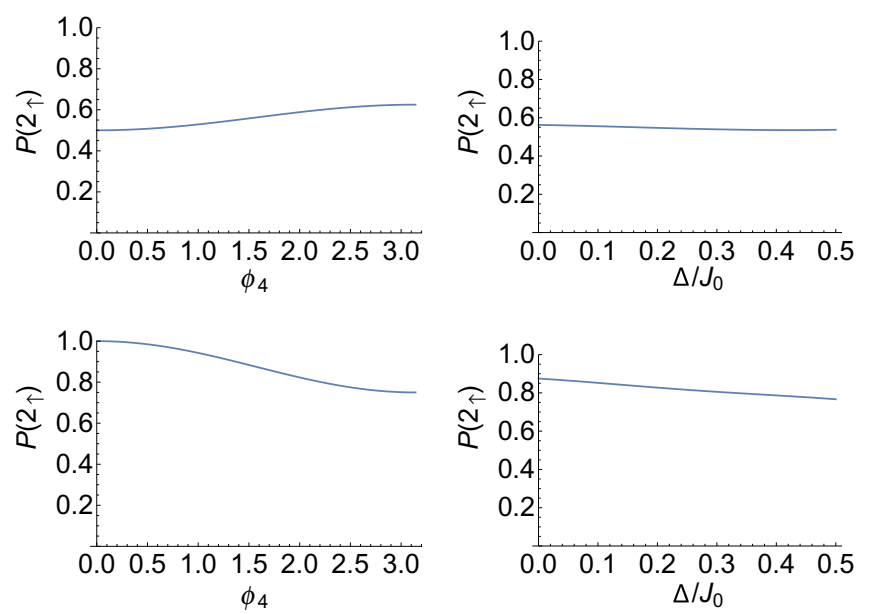

FIG. 5. The probability that dot 2 will be measured in spin state $\uparrow$ after a sequence of gates is applied to the initial state $\left|\uparrow_{+} \downarrow_{+} \uparrow_{+} \downarrow_{+}\right\rangle$, with $\phi_{1}=\phi_{2}=\phi_{3}=0$. Top left: the gate sequence is given by Eq. (14) and $\Delta=0$. Top right: the gate sequence is given by Eq. (14) and $\phi_{4}=\pi / 2$. Bottom left: the gate sequence is given by Eq. (17) and $\Delta=0$. Bottom right: the gate sequence is given by Eq. (17) and $\phi_{4}=\pi / 2$.

We also plot the measurement probability as a function of $\Delta$, showing that the effective decoherence increases as $\Delta$ increases, provided that $\Delta$ still stays within the regime $\Delta \ll J_{0}$.

\section{CONCLUSION}

We use an effective Hubbard model to investigate the effects of valley states in silicon quantum dots in the context of exchange gate operations in multiqubit systems at zero temperature. We first consider a system of two exchange-coupled quantum dots and determine the eigenstates and energies for both the singlet and triplet spin configurations to leading order in $t_{c}^{2} / U$. We consider the limits where $\Delta \gg J_{0}$ and $\Delta \ll J_{0}$ and find that in both cases, the singlet and triplet spin configurations share the same eigenstates, though their energies differ. When the valley splitting is large, any state not initialized to valley eigenstates will lead to spin-valley entanglement as the system evolves. However, as long as the valley splitting is large and the system is initialized to the valley ground state, the evolution of the system will not be affected to leading order in $\Delta / J_{0}$. Thus valley degrees of freedom are not problematic for exchange gate operations as long as the valley splitting $\Delta$ is sufficiently large. For small valley splitting or completely degenerate valley states, spin-valley entanglement will arise any time the electrons are not initialized to the same valley state. Without the ability to perform valley state measurements, spin-valley entanglement will obfuscate any information stored in the electron spin states, making it impossible to perform quantum computations in such a state. For a two-qubit system with $\Delta \ll J_{0}$, if all spins are initialized in $z$ eigenstates and measured in the $z$ basis, then the resulting measurement probabilities will be the same as for an ideal single-valley system. However, this does not extend to systems with more qubits, as spin-valley entanglement can introduce errors despite states being initialized and measured in the $z$ basis. This means that two-qubit gate fidelities measured by performing a single gate in a twoqubit system will give deceptively high-fidelity results, because the presence of valley states does not affect the fidelity measurement even though it can affect operations in systems with more qubits. To demonstrate robustness to spin-valley entanglement without the ability to measure valley states themselves requires a longer series of gates in a system with more qubits. We emphasize that our use of the Hubbard model is not an approximation here, since the coupled spin qubit system is indeed equivalent to the Hubbard model, the Hubbard interaction $U$ being simply related to the exchange coupling $J_{0}$ between the dots through $U=t_{c}^{2} / J_{0}$, where $t_{c}$ is the interdot hopping energy from wave-function overlap. Our fundamental finding of an apparent quantum leakage (or effective decoherence) due to spin-valley entanglement is also independent of additional complications arising from varying exchange couplings and/or interdot hopping through the circuit - all they do is to complicate the expressions for the leakage but the basic physics of spin-valley entanglement remains the same.

We note that our considerations on spin-valley entanglement apply equally well to all-exchange gate operations considered in Ref. [38] - any time the interdot exchange coupling is used to carry out gate operations, the valleyspin entanglement (and potential decoherence to valley states) studied in this work becomes relevant. Right now, the Si qubit platforms have rather small exchange coupling and the problem discussed in this work is most likely not crucial to the current generation of few qubit systems with rather small exchange coupling values. But faster gate operations in multiqubit circuits will necessitate larger exchange coupling strength in the future, making our dynamical consideration relevant, as one must ensure that not only is the valley splitting much larger than the electron temperature in the qubits but it is also much larger than the interdot exchange coupling used in the gate operations.

We now critically discuss some aspects and approximations of our theory. First and foremost, the subtle physics of valley splitting on two-qubit operations that we point out in this work is completely independent of the already well-known problem associated with valley occupancy in Si qubits, which already necessitates having large valley splittings compared with temperature $T$. For our physics, the valley splitting must be much larger than the interqubit exchange coupling $J_{0}$. Since the current experimentally relevant values of $J$ for existing $\mathrm{Si}$ qubits are rather small, often comparable to (or even smaller than) $T$ itself (although $T$ and $J$ are completely 
independent parameters and have nothing to do with each other), it may seem that our theory is not particularly germane to the current experimental Si qubit activity, since the condition for the valley splitting $\Delta$ being larger than $J$ appears to be automatically satisfied in current devices by having splitting larger than temperature. This conclusion, however, may be misleading for two reasons. First, the functional dependencies of "errors" introduced by valley spitting in the two situations are very different: temperature enters exponentially through the thermal occupancy factor $e^{-\Delta / T}$, adversely affecting single-qubit operations, whereas, by contrast, as we show in the current work, the two-qubit fidelity is adversely affected algebraically by $J_{0} / \Delta$. Thus, the same value of $\Delta$ in a device may suffice to satisfy the condition $e^{-\Delta / T} \ll 1$ but completely fail to satisfy $J_{0} / \Delta \ll 1$. This means that for a given set of values of $\Delta, T$, and $J_{0}$ for a device, it is possible for the valley splitting to be large for high-fidelity single-qubit operations (i.e., exponentially low thermal occupancy) and low-fidelity two-qubit operations (as described in our current work) because of not-so-small values of $J_{0} / \Delta$. Considering a device with $T=25 \mathrm{mK}, \Delta=300 \mathrm{mK}$, and $J_{0}=10 \mathrm{mK}$, which are typical numbers, we obtain $e^{-\Delta / T} \sim 6 \times 10^{-6}$ and $J_{0} / \Delta \sim 3 \times 10^{-2}$, implying that while valley-splitting-induced single-qubit thermal occupancy errors are well below the quantum error-correction threshold, the corresponding two-qubit errors discussed in the current work are not small enough, being 4 orders of magnitude larger than the one-qubit error. The second reason is that in a many-qubit device, it is always possible to have a particular qubit with a valley splitting that is not very large (particularly since there is no automatic way of guaranteeing large valley splitting in a device), which, as we establish in the current work, will lead to two-qubit errors that will not directly show up in two-qubit operations. Thus, there is simply no way to ensure that the problem we describe in the current work is not lurking in a multiqubit device without going through the full multiqubit characterization, as we discuss in this paper. We also emphasize that the eventual Si quantum computer will have millions of qubits and that the problem we have discovered may become crucial in such a large device. It is much better to be aware of this problem now, so that the condition $\Delta \gg J_{0}$ is satisfied everywhere in order to avoid unknown two-qubit errors.

The second issue that we discuss is our use of the Hubbard model in Eq. (1), which may appear to be a drastic approximation, since real Si qubits do not obey the Hubbard model precisely in quantitative terms - the applicability or not of the Hubbard model for Si qubits has been discussed in Ref. [35]. This is, however, not a limitation of the theory at all, since the only aspect of the Hubbard model relevant for our theory is the fact that it leads to an interdot exchange coupling $\left(J_{0}=4 t_{c}^{2} / U\right)$ and, in the end, $J_{0}$ is the parameter to be compared with the valley splitting $\Delta$ to decide whether one is in the large$\left(\Delta \gg J_{0}\right)$ or small-valley-splitting regime. The Hubbard model allows a straightforward transformation between electrons in the quantum dots and localized Heisenberg spins as two-level qubit systems and it enables a full analytical treatment of the problem. The fully analytical nature of our theory is a particularly attractive feature, since it makes the theory universally applicable to all Si devices in different laboratories - all one needs to do is to carry out numerical simulations for the specific Si structure to obtain the interqubit exchange coupling. Thus, the Hubbard model description makes our theory universal and specific devices can be adapted to the theory by calculating the exchange coupling realistically, which must be done individually for each device.

Although our focus in this work is on spin-valley entanglement leading to a rather subtle (and difficult-toobserve) "valley-induced leakage" during multiqubit gate operations for small valley splittings, there is an issue for large valley splitting that is also implicit (but not emphasized here) in our work. Even for large valley splitting, the exchange gate operations are adversely affected by an effective valley-induced "renormalization" of the exchange coupling. In our work, we find that in the largevalley-splitting limit, the effective exchange coupling $J_{+}=4\left|t_{+}\right|^{2} / U$, which is proportional to $\left|1+e^{i \delta \phi}\right|^{2}$. This implies that $J_{+}$vanishes as $\delta \phi$ goes to $\pi$. However, we do not specifically write out or discuss this result in our paper, as our main focus is spin-valley entanglement in the small-valley-splitting case. We note that this modification of the effective exchange coupling, happening for any valley splitting, is included throughout our theoretical work, but is not emphasized since it does not lead to a leakage and is not a spin-valley entanglement effect. Having a phase difference close to $\pi$ could thus strongly suppress the exchange coupling, but this is a separate issue compared to whether or not leakage and/or entanglement occur(s), which is the main question that we address. For the most part, we allow the phase difference to be arbitrary in our work, although in a few specific examples we set it to 0 or $\pi$ for the sake of simplicity. We do not look into the resulting statistics of random phase differences, though that could be a potentially interesting direction for future work. Thus, valley splitting may in fact be relevant for multiqubit operations even when it is large but does not necessarily cause leakage.

The most obvious mitigation of the valley-degeneracyinduced exchange operation problem described in this work is to have the valley splitting being much larger than the exchange coupling and initialization of all dots in the lower-energy ground valleys, which is guaranteed if the exchange coupling strength is very small. But for faster exchange gate operations with larger exchange coupling, other mitigation measures may be necessary. One possibility is to carry out measurements as a function 
of different values of $J$, enabling a characterization of whether or not two-qubit exchange operations are problematic because of small valley splitting. Spin-valley entanglement could be directly observed in a two-qubit system if sufficient control over the exchange interaction is available such that a time-dependent pulse $J(t)$ can be performed where some part of the pulse $J(t)$ is much smaller than the valley splitting. Another possibility is measurements using different basis states, which may be carried out under some type of random benchmarking protocol. The addition of other terms to the Hamiltonian, such as a magnetic field gradient, may also allow potential spinvalley entanglement to be observed in a two-qubit system with large and constant $J$ [39]. Our main purpose in this paper is to alert the community to the fact that although it is absolutely necessary for valley splitting to be much larger than the temperature in order to make sure that the thermal occupancy of higher valley states does not compromise the qubit fidelity, this by itself is not sufficient, as one must also take the relative magnitude of valley splitting and exchange coupling into account in order to ensure high-fidelity exchange gate operations even at zero temperature.

In this context, it is encouraging that recent experimental work has had considerable success in consistently achieving large valley splittings [40,41], although in a many-qubit circuit, whether or not each qubit has large splittings still must be determined on an individual basis, which makes it a challenge to scale up to a large qubit system, which is considered one of the key advantages of $\mathrm{Si}$ spin qubits. We conclude by emphasizing that although our work may not be of immediate relevance to the existing experimental Si qubit circuits, mainly because of the fewqubit (between one and four) nature of the current devices and their rather low values of exchange coupling, eventual Si circuits that would hopefully compete with the manyqubit superconducting transmon circuits some day in the future would have to incorporate the potential problem that we have discovered.

\section{ACKNOWLEDGMENTS}

We thank Professors Xuedong Hu and Jason Kestner for useful correspondence on the work. This work is supported by the Laboratory for Physical Sciences.

\section{APPENDIX: DERIVATION OF EQS. (3) AND (6)}

In this appendix, we give the detailed derivation of Eqs. (3) and (6), beginning from the Hamiltonian $H$ given by Eq. (1).

The Hubbard Hamiltonian $H$ acts on a vector space of eight single-particle states, corresponding to every combination of choices for $\operatorname{dot} j=|1\rangle$ or $|2\rangle$, valley state $v=|+\rangle$ or $|-\rangle$, and spin $s=|\uparrow\rangle$ or $|\downarrow\rangle$. We consider two electrons in this system, corresponding to $\left(\begin{array}{l}8 \\ 2\end{array}\right)=28$ two-particle states. Because $H$ commutes with the spin operators $S^{2}$ and $S_{z}$, spin is conserved and $H$ can be divided into the following four noninteracting blocks corresponding to the total spin of the system: the singlet subspace, which consists of ten states; and three triplet subspaces with $S_{z}=-1,0$, or 1 , each of which consisting of six states. The three triplet subspaces are identical up to the value of $S_{z}$, so we focus only on one of them (where $S_{z}=0$ ). The six triplet states are as follows:

$$
\begin{aligned}
& \left|1_{+} 1_{-}\right\rangle=\frac{c_{1,+, \uparrow}^{\dagger} c_{1,-, \downarrow}^{\dagger}+c_{1,+, \downarrow}^{\dagger} c_{1,-, \uparrow}^{\dagger}}{\sqrt{2}}|0\rangle, \\
& \left|1_{+} 2_{+}\right\rangle=\frac{c_{1,+, \uparrow}^{\dagger} c_{2,+, \downarrow}^{\dagger}+c_{1,+, \downarrow}^{\dagger} c_{2,+, \uparrow}^{\dagger}}{\sqrt{2}}|0\rangle, \\
& \left|1_{+} 2_{-}\right\rangle=\frac{c_{1,+, \uparrow}^{\dagger} c_{2,-, \downarrow}^{\dagger}+c_{1,+, \downarrow}^{\dagger} c_{2,-, \uparrow}^{\dagger}}{\sqrt{2}}|0\rangle, \\
& \left|1_{-} 2_{+}\right\rangle=\frac{c_{1,-, \uparrow}^{\dagger} c_{2,+, \downarrow}^{\dagger}+c_{1,-, \downarrow}^{\dagger} c_{2,+, \uparrow}^{\dagger}}{\sqrt{2}}|0\rangle, \\
& \left|1_{-} 2_{-}\right\rangle=\frac{c_{1,-, \uparrow}^{\dagger} c_{2,-, \downarrow}^{\dagger}+c_{1,-, \downarrow}^{\dagger} c_{2,-, \uparrow}^{\dagger}}{\sqrt{2}}|0\rangle, \\
& \left|2_{+} 2_{-}\right\rangle=\frac{c_{2,+, \uparrow}^{\dagger} c_{2,-, \downarrow}^{\dagger}+c_{2,+, \downarrow}^{\dagger} c_{2,-, \uparrow}^{\dagger}}{\sqrt{2}}|0\rangle,
\end{aligned}
$$

where $|0\rangle$ is the "vacuum" state corresponding to the system with no electrons in either dot. Writing the Hamiltonian $H$ in the basis given by Eq. (A1) yields the following:

$$
H_{T}=\left(\begin{array}{cccccc}
U+\epsilon & t_{-} & t_{+} & -t_{+} & -t_{-} & 0 \\
t_{-}^{*} & \Delta_{1}+\Delta_{2} & 0 & 0 & 0 & -t_{-} \\
t_{+}^{*} & 0 & \Delta_{1}-\Delta_{2} & 0 & 0 & t_{+} \\
-t_{+}^{*} & 0 & 0 & -\Delta_{1}+\Delta_{2} & 0 & -t_{+} \\
-t_{-}^{*} & 0 & 0 & 0 & -\Delta_{1}-\Delta_{2} & t_{-} \\
0 & -t_{-}^{*} & t_{+}^{*} & -t_{+}^{*} & t_{-}^{*} & U-\epsilon
\end{array}\right)
$$


Note that matrix elements corresponding to an electron moving from one valley state to the other in the same dot are 0 , since the Hamiltonian is written in terms of the valley eigenstates $|+\rangle$ and $|-\rangle$. Some tunneling-matrix elements such as $\left|1_{+} 1_{-}\right\rangle \rightarrow\left|1_{-} 2_{-}\right\rangle$contain an additional minus sign due to Fermi statistics, since the particle that does not tunnel (in this case, $1_{-}$) switches from the second position to the first position in the state definition (compare this to the matrix element $\left|1_{+} 1_{-}\right\rangle \rightarrow\left|1_{+} 2_{+}\right\rangle$, where $1_{+}$is in the first position in both states). Switching the position corresponds to commuting two creation operators, which produces a minus sign.
The $\left|1_{+} 1_{-}\right\rangle$and $\left|2_{+} 2_{-}\right\rangle$states contain two electrons in a single dot, which cause the states to have a much higher energy due to the on-site Coulomb interaction energy $U$. Thus these states will not directly affect the system dynamics; however, there will still be an indirect perturbative effect on the lower-energy states and energies. To determine this effect, we perform a routine perturbationtheory calculation with the unperturbed Hamiltonian $H_{0}=\operatorname{diag}(U, 0,0,0,0, U)$ and the perturbation $H^{\prime}$ consisting of the rest of the matrix elements in Eq. (A2). Assuming that $U \gg t_{c}, \Delta_{j}, \epsilon$, the first-order correction in $U^{-1}$ to the Hamiltonian is given by

$$
H_{T}=\left(\begin{array}{cccc}
\Delta_{1}+\Delta_{2}-\frac{2\left|t_{-}\right|^{2}}{U} & \frac{t_{-} t_{+}^{*}-t_{+} t_{-}^{*}}{U} & \frac{t_{+} t_{-}^{*}-t_{-} t_{+}^{*}}{U} & \frac{2\left|t_{-}\right|^{2}}{U} \\
\frac{t_{+} t_{-}^{*}-t_{-} t_{+}^{*}}{U} & \Delta_{1}-\Delta_{2}-\frac{2\left|t_{+}\right|^{2}}{U} & \frac{2\left|t_{+}\right|^{2}}{U} & \frac{t_{-} t_{+}^{*}-t_{+} t_{-}^{*}}{U} \\
\frac{t_{-} t_{+}^{*}-t_{+} t_{-}^{*}}{U} & \frac{2\left|t_{+}\right|^{2}}{U} & -\Delta_{1}+\Delta_{2}-\frac{2\left|t_{+}\right|^{2}}{U} & \frac{t_{+} t_{-}^{*}-t_{-} t_{+}^{*}}{U} \\
\frac{2\left|t_{-}\right|^{2}}{U} & \frac{t_{+} t_{-}^{*}-t_{-} t_{+}^{*}}{U} & \frac{t_{-} t_{+}^{*}-t_{+} t_{-}^{*}}{U} & -\Delta_{1}-\Delta_{2}-\frac{2\left|t_{-}\right|^{2}}{U}
\end{array}\right),
$$

where only the four low-energy states are shown.

A similar process is carried out for the singlet space, where there are ten states in total:

$$
\begin{aligned}
& \left|1_{+} 1_{-}\right\rangle=\frac{c_{1,+, \uparrow}^{\dagger} c_{1,-, \downarrow}^{\dagger}-c_{1,+, \downarrow}^{\dagger} c_{1,-, \uparrow}^{\dagger}}{\sqrt{2}}|0\rangle, \\
& \left|1_{+} 2_{+}\right\rangle=\frac{c_{1,+, \uparrow}^{\dagger} c_{2,+, \downarrow}^{\dagger}-c_{1,+, \downarrow}^{\dagger} c_{2,+, \uparrow}^{\dagger}}{\sqrt{2}}|0\rangle, \\
& \left|1_{+} 2_{-}\right\rangle=\frac{c_{1,+, \uparrow}^{\dagger} c_{2,-, \downarrow}^{\dagger}-c_{1,+, \downarrow}^{\dagger} c_{2,-, \uparrow}^{\dagger}}{\sqrt{2}}|0\rangle, \\
& \left|1_{-} 2_{+}\right\rangle=\frac{c_{1,-, \uparrow}^{\dagger} c_{2,+, \downarrow}^{\dagger}-c_{1,-, \downarrow}^{\dagger} c_{2,+, \uparrow}^{\dagger}}{\sqrt{2}}|0\rangle, \\
& \left|1_{-} 2_{-}\right\rangle=\frac{c_{1,-, \uparrow}^{\dagger} c_{2,-, \downarrow}^{\dagger}-c_{1,-, \downarrow}^{\dagger} c_{2,-, \uparrow}^{\dagger}}{\sqrt{2}}|0\rangle, \\
& \left|2_{+} 2_{-}\right\rangle=\frac{c_{2,+, \uparrow}^{\dagger} c_{2,-, \downarrow}^{\dagger}-c_{2,+, \downarrow}^{\dagger} c_{2,-, \uparrow}^{\dagger}}{\sqrt{2}}|0\rangle, \\
& \left|1_{+1 \downarrow}\right\rangle=c_{1,+, \uparrow}^{\dagger} c_{1,+, \downarrow}^{\dagger}|0\rangle, \\
& \left|1_{-1 \downarrow}\right\rangle=c_{1,-, \uparrow}^{\dagger} c_{1,-, \downarrow}^{\dagger}|0\rangle, \\
& \left|2_{+1 \downarrow}\right\rangle=c_{2,+, \uparrow}^{\dagger} c_{2,+, \downarrow}^{\dagger}|0\rangle, \\
& \left|2_{-1 \downarrow}\right\rangle=c_{2,-, \uparrow}^{\dagger} c_{2,-, \downarrow}^{\dagger}|0\rangle .
\end{aligned}
$$


Writing $H$ in this basis yields

$$
H_{S}=\left(\begin{array}{cccccccccc}
U+\epsilon & t_{-} & t_{+} & t_{+} & t_{-} & 0 & 0 & 0 & 0 & 0 \\
t_{-}^{*} & \Delta_{1}+\Delta_{2} & 0 & 0 & 0 & t_{-} & \sqrt{2} t_{+}^{*} & 0 & \sqrt{2} t_{+} & 0 \\
t_{+}^{*} & 0 & \Delta_{1}-\Delta_{2} & 0 & 0 & t_{+} & \sqrt{2} t_{-}^{*} & 0 & 0 & \sqrt{2} t_{-} \\
t_{+}^{*} & 0 & 0 & -\Delta_{1}+\Delta_{2} & 0 & t_{+} & 0 & \sqrt{2} t_{-}^{*} & \sqrt{2} t_{-} & 0 \\
t_{-}^{*} & 0 & 0 & 0 & -\Delta_{1}-\Delta_{2} & t_{-} & 0 & \sqrt{2} t_{+}^{*} & 0 & \sqrt{2} t_{+} \\
0 & t_{-}^{*} & t_{+}^{*} & t_{+}^{*} & t_{-}^{*} & U-\epsilon & 0 & 0 & 0 & 0 \\
0 & \sqrt{2} t_{+} & \sqrt{2} t_{-} & 0 & 0 & 0 & U+\epsilon+2 \Delta_{1} & 0 & 0 & 0 \\
0 & 0 & 0 & \sqrt{2} t_{-} & \sqrt{2} t_{+} & 0 & 0 & U+\epsilon-2 \Delta_{1} & 0 & 0 \\
0 & \sqrt{2} t_{+}^{*} & 0 & \sqrt{2} t_{-}^{*} & 0 & 0 & 0 & 0 & U-\epsilon+2 \Delta_{2} & 0 \\
0 & 0 & \sqrt{2} t_{-}^{*} & 0 & \sqrt{2} t_{+}^{*} & 0 & 0 & 0 & 0 & U-\epsilon-2 \Delta_{2}
\end{array}\right) .
$$

Here, the minus sign due to Fermi statistics is canceled by a second minus sign arising from swapping the spins of a singlet state, making all tunneling-matrix elements positive. Again performing a perturbation theory expansion produces the following Hamiltonian:

$$
H_{S}=\left(\begin{array}{cccc}
\Delta_{1}+\Delta_{2}-\frac{2\left|t_{-}\right|^{2}+4\left|t_{+}\right|^{2}}{U} & -\frac{2 t_{-} t_{+}^{*}}{U} & -\frac{2 t_{+} t_{-}^{*}}{U} & -\frac{2\left|t_{-}\right|^{2}}{U} \\
-\frac{2 t_{+} t_{-}^{*}}{U} & \Delta_{1}-\Delta_{2}-\frac{2\left|t_{+}\right|^{2}+4\left|t_{-}\right|^{2}}{U} & -\frac{2\left|t_{+}\right|^{2}}{U} & -\frac{2 t_{-} t_{+}^{*}}{U} \\
-\frac{2 t_{-} t_{+}^{*}}{U} & -\frac{2\left|t_{+}\right|^{2}}{U} & -\Delta_{1}+\Delta_{2}-\frac{2\left|t_{+}\right|^{2}+4\left|t_{-}\right|^{2}}{U} & -\frac{2 t_{+} t_{-}^{*}}{U} \\
-\frac{2\left|t_{-}\right|^{2}}{U} & -\frac{2 t_{+} t_{-}^{*}}{U} & -\frac{2 t_{-} t_{+}^{*}}{U} & -\Delta_{1}-\Delta_{2}-\frac{2\left|t_{-}\right|^{2}+4\left|t_{+}\right|^{2}}{U}
\end{array}\right) .
$$

[1] E. Kawakami, P. Scarlino, D. R. Ward, F. R. Braakman, D. E. Savage, M. G. Lagally, M. Friesen, S. N. Coppersmith, M. A. Eriksson, and L. M. K. Vandersypen, Electrical control of a long-lived spin qubit in a Si/SiGe quantum dot, Nat. Nanotechnol. 9, 666 (2014).

[2] M. Veldhorst, C. H. Yang, J. C. C. Hwang, W. Huang, J. P. Dehollain, J. T. Muhonen, S. Simmons, A. Laucht, F. E. Hudson, K. M. Itoh, et al., A two-qubit logic gate in silicon, Nature 526, 410 (2015).

[3] R. Maurand, X. Jehl, D. Kotekar-Patil, A. Corna, H. Bohuslavskyi, R. Laviéville, L. Hutin, S. Barraud, M. Vinet, M. Sanquer, et al., A CMOS silicon spin qubit, Nat. Commun. 7, 13575 (2016).

[4] D. M. Zajac, T. M. Hazard, X. Mi, E. Nielsen, and J. R. Petta, Scalable Gate Architecture for a One-Dimensional Array of Semiconductor Spin Qubits, Phys. Rev. Appl. 6, 054013 (2016).

[5] M. A. Fogarty, K. W. Chan, B. Hensen, W. Huang, T. Tanttu, C. H. Yang, A. Laucht, M. Veldhorst, F. E. Hudson, K. M. Itoh, et al., Integrated silicon qubit platform with single-spin addressability, exchange control and single-shot singlet-triplet readout, Nat. Commun. 9, 4370 (2018).

[6] T. F. Watson, S. G. J. Philips, E. Kawakami, D. R. Ward, P. Scarlino, M. Veldhorst, D. E. Savage, M. G. Lagally, M. Friesen, S. N. Coppersmith, et al., A programmable twoqubit quantum processor in silicon, Nature 555, 633 (2018).

[7] D. M. Zajac, A. J. Sigillito, M. Russ, F. Borjans, J. M. Taylor, G. Burkard, and J. R. Petta, Resonantly driven CNOT gate for electron spins, Science 359, 439 (2018).

[8] W. Huang, C. H. Yang, K. W. Chan, T. Tanttu, B. Hensen, R. C. C. Leon, M. A. Fogarty, J. C. C. Hwang, F. E. Hudson, K. M. Itoh, et al., Fidelity benchmarks for two-qubit gates in silicon, Nature 569, 532 (2019).

[9] A. R. Mills, D. M. Zajac, M. J. Gullans, F. J. Schupp, T. M. Hazard, and J. R. Petta, Shuttling a single charge across a one-dimensional array of silicon quantum dots, Nat. Commun. 10, 1063 (2019).

[10] A. J. Sigillito, M. J. Gullans, L. F. Edge, M. Borselli, and J. R. Petta, Coherent transfer of quantum information in a silicon double quantum dot using resonant SWAP gates, npj Quantum Inf. 5, 110 (2019).

[11] X. Xue, T. F. Watson, J. Helsen, D. R. Ward, D. E. Savage, M. G. Lagally, S. N. Coppersmith, M. A. Eriksson, S. Wehner, and L. M. K. Vandersypen, Benchmarking Gate Fidelities in a Si/SiGe Two-Qubit Device, Phys. Rev. X 9, 021011 (2019).

[12] R. Zhao, T. Tanttu, K. Y. Tan, B. Hensen, K. W. Chan, J. C. C. Hwang, R. C. C. Leon, C. H. Yang, W. Gilbert, F. E. Hudson, et al., Single-spin qubits in isotopically enriched silicon at low magnetic field, Nat. Commun. 10, 5500 (2019).

[13] F. Borjans, X. G. Croot, X. Mi, M. J. Gullans, and J. R. Petta, Resonant microwave-mediated interactions between distant electron spins, Nature 577, 195 (2020). 
[14] L. Petit, H. G. J. Eenink, M. Russ, W. I. L. Lawrie, N. W. Hendrickx, S. G. J. Philips, J. S. Clarke, L. M. K. Vandersypen, and M. Veldhorst, Universal quantum logic in hot silicon qubits, Nature 580, 355 (2020).

[15] X. Xue, B. D'Anjou, T. F. Watson, D. R. Ward, D. E. Savage, M. G. Lagally, M. Friesen, S. N. Coppersmith, M. A. Eriksson, W. A. Coish, et al., Repetitive Quantum Nondemolition Measurement and Soft Decoding of a Silicon Spin Qubit, Phys. Rev. X 10, 021006 (2020).

[16] C. H. Yang, R. C. C. Leon, J. C. C. Hwang, A. Saraiva, T. Tanttu, W. Huang, J. Camirand Lemyre, K. W. Chan, K. Y. Tan, F. E. Hudson, et al., Operation of a silicon quantum processor unit cell above one kelvin, Nature 580, 350 (2020).

[17] P. W. Shor, Polynomial-time algorithms for prime factorization and discrete logarithms on a quantum computer, SIAM J. Comput. 26, 1484 (1997).

[18] W. M. Witzel, M. S. Carroll, A. Morello, L. Cywiński, and S. Das Sarma, Electron Spin Decoherence in IsotopeEnriched Silicon, Phys. Rev. Lett. 105, 187602 (2010).

[19] A. L. Saraiva, M. J. Calderón, X. Hu, S. Das Sarma, and B. Koiller, Physical mechanisms of interface-mediated intervalley coupling in Si, Phys. Rev. B 80, 081305 (2009).

[20] A. L. Saraiva, M. J. Calderón, R. B. Capaz, X. Hu, S. Das Sarma, and B. Koiller, Intervalley coupling for interfacebound electrons in silicon: An effective mass study, Phys. Rev. B 84, 155320 (2011).

[21] P. Recher, J. Nilsson, G. Burkard, and B. Trauzettel, Bound states and magnetic field induced valley splitting in gatetunable graphene quantum dots, Phys. Rev. B 79, 085407 (2009).

[22] D. Culcer, A. L. Saraiva, B. Koiller, X. Hu, and S. Das Sarma, Valley-Based Noise-Resistant Quantum Computation Using Si Quantum Dots, Phys. Rev. Lett. 108, 126804 (2012).

[23] N. Rohling and G. Burkard, Universal quantum computing with spin and valley states, New J. Phys. 14, 083008 (2012).

[24] J. Corrigan, J. P. Dodson, H. E. Ercan, J. C. Abadillo-Uriel, B. Thorgrimsson, T. J. Knapp, N. Holman, T. McJunkin, S. F. Neyens, E. R. MacQuarrie, et al., Coherent control and spectroscopy of a semiconductor quantum dot Wigner molecule, ArXiv:2009.13572 (2020).

[25] J. P. Dodson, H. E. Ercan, J. Corrigan, M. Losert, N. Holman, T. McJunkin, L. F. Edge, M. Friesen, S. N. Coppersmith, and M. A. Eriksson, How valley-orbit states in silicon quantum dots probe quantum well interfaces, ArXiv:2103.14702 (2021).

[26] B. P. Wuetz, M. P. Losert, A. Tosato, M. Lodari, P. L. Bavdaz, L. Stehouwer, P. Amin, J. S. Clarke, S. N. Coppersmith, A. Sammak, et al., Effect of Quantum Hall Edge Strips on Valley Splitting in Silicon Quantum Wells, Phys. Rev. Lett. 125, 186801 (2020).
[27] Y.-P. Shim, R. Ruskov, H. M. Hurst, and C. Tahan, Induced quantum dot probe for material characterization, Appl. Phys. Lett. 114, 152105 (2019).

[28] S. W. Oh, A. O. Denisov, P. Chen, and J. R. Petta, Cryogen-free scanning gate microscope for the characterization of $\mathrm{Si} / \mathrm{Si}_{0.7} \mathrm{Ge}_{0.3}$ quantum devices at milli-Kelvin temperatures, ArXiv:2105.05684 (2021).

[29] Z. Jiang, N. Kharche, T. Boykin, and G. Klimeck, Effects of interface disorder on valley splitting in $\mathrm{SiGe} / \mathrm{Si} / \mathrm{SiGe}$ quantum wells, Appl. Phys. Lett. 100, 103502 (2012).

[30] B. Tariq and X. Hu, Effects of interface steps on the valleyorbit coupling in a Si/SiGe quantum dot, Phys. Rev. B 100, 125309 (2019).

[31] C. H. Yang, W. H. Lim, N. S. Lai, A. Rossi, A. Morello, and A. S. Dzurak, Orbital and valley state spectra of a few-electron silicon quantum dot, Phys. Rev. B 86, 115319 (2012).

[32] A. Sigillito, J. Loy, D. Zajac, M. Gullans, L. Edge, and J. Petta, Site-Selective Quantum Control in an Isotopically Enriched $28^{\mathrm{Si}} / \mathrm{Si}_{0.7} \mathrm{Ge}_{0.3}$ Quadruple Quantum Dot Phys. Rev. Appl. 11, 061006 (2019).

[33] S. F. Neyens, R. H. Foote, B. Thorgrimsson, T. J. Knapp, T. McJunkin, L. M. K. Vandersypen, P. Amin, N. K. Thomas, J. S. Clarke, D. E. Savage, et al., The critical role of substrate disorder in valley splitting in Si quantum wells, Appl. Phys. Lett. 112, 243107 (2018).

[34] F. Borjans, X. Zhang, X. Mi, G. Cheng, N. Yao, C. Jackson, L. Edge, and J. Petta, Probing the variation of the intervalley tunnel coupling in a silicon triple quantum dot, PRX Quantum 2, 020309 (2021).

[35] S. Das Sarma, X. Wang, and S. Yang, Hubbard model description of silicon spin qubits: Charge stability diagram and tunnel coupling in Si double quantum dots, Phys. Rev. B 83, 235314 (2011).

[36] S. Yang, X. Wang, and S. Das Sarma, Generic Hubbard model description of semiconductor quantum-dot spin qubits, Phys. Rev. B 83, 161301 (2011).

[37] C. A. Stafford and S. Das Sarma, Collective Coulomb Blockade in an Array of Quantum Dots: A Mott-Hubbard Approach, Phys. Rev. Lett. 72, 3590 (1994).

[38] D. P. DiVincenzo, D. Bacon, J. Kempe, G. Burkard, and K. B. Whaley, Universal quantum computation with the exchange interaction, Nature 408, 339 (2000).

[39] J. Kestner, Private communication.

[40] C. H. Yang, A. Rossi, R. Ruskov, N. S. Lai, F. A. Mohiyaddin, S. Lee, C. Tahan, G. Klimeck, A. Morello, and A. S. Dzurak, Spin-valley lifetimes in a silicon quantum dot with tunable valley splitting, Nat. Commun. 4, 2069 (2013).

[41] J. K. Gamble, P. Harvey-Collard, N. T. Jacobson, A. D. Baczewski, E. Nielsen, L. Maurer, I. Montaño, M. Rudolph, M. S. Carroll, C. H. Yang, et al., Valley splitting of singleelectron Si MOS quantum dots, Appl. Phys. Lett. 109, 253101 (2016). 